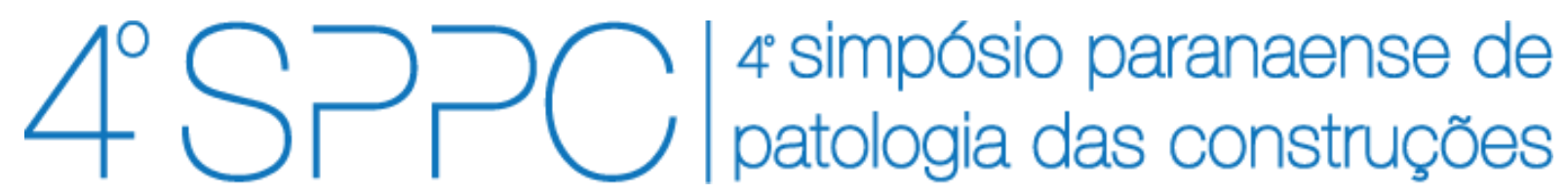

ISSN 2526-7248 artigo n. 4SPPC142, pp. 381-390, 2019

\title{
Desempenho mecânico e físico de concretos com adição de nanosílica e nanoalumina coloidais
}

\author{
Orellana, Juan Pedro Luvizotti'; Bragança, Mariana d'Orey Gaivão Portella²; \\ Portella, Kleber Franke ${ }^{3}$; Alberti, Emerson Luís ${ }^{4}$ \\ ${ }^{1}$ Mestrando, Universidade Federal do Paraná (PPGECC)/Lactec, \\ jpedrool.93@gmail.com \\ 2 Doutora, Universidade Federal do Paraná/Lactec, mariana.portella@lactec.org.br \\ 3 Doutor, Universidade Federal do Paraná/Lactec, portella@lactec.org.br \\ ${ }^{4}$ Pesquisador, Centrais Elétricas do Rio Jordão, emerson@elejor.com.br
}

\begin{abstract}
Resumo: Na construção civil, vários materiais com escala nano estão sendo estudados como adição ao concreto, com a finalidade de analisar, principalmente, as propriedades mecânicas e de durabilidade do mesmo. Entre estes, encontram-se os óxidos nanométricos, como é o caso do nano- $\mathrm{SiO}_{2}$ (nanosílica) e o nano- $\mathrm{Al}_{2} \mathrm{O}_{3}$ (nanoalumina). Assim, o objetivo deste trabalho foi avaliar as propriedades mecânicas e físicas de concretos com adição de 1,5\% destes dois nanomateriais. Foram constatadas melhorias nas propriedades mecânicas nos concretos com as duas nanoadições e, em destaque, para a nanosílica. No que diz respeito à absorção de água, foi observada uma redução dos seus valores em cerca de $24 \%$ para os traços com estes materiais, o que pode ter sido resultante de uma redução da porosidade do concreto. Nos resultados do ensaio de absorção capilar de água foram obtidos aumentos nos valores para esses traços, o que pode indicar uma redução do raio médio dos poros, favorecendo à durabilidade do concreto.
\end{abstract}

Palavras-chave: Nanomateriais, desempenho mecânico, absorção de água.

\begin{abstract}
In the civil construction, several nanoscale materials are being studied as an addition to concrete, with the purpose of analyzing, mainly, the mechanical properties and durability of the same. Among these are the nanometric oxides, as is the case of nano- $\mathrm{SiO}_{2}$ (nanosilica) and nano- $\mathrm{Al}_{2} \mathrm{O}_{3}$ (nanoalumina). Thus, the objective of this work was to evaluate the mechanical and physical properties of concrete with addition of $1.5 \%$ of these two nanomaterials. An improvement was observed in the mechanical properties of the concretes with the two nanoaditions, and, in particular, for the nanosilica. With regard to water absorption, a reduction of their values was observed in about $24 \%$ for the traces with these materials, which may have been resulting from a reduction of the porosity of the concrete. In the results of the capillary water absorption test, increases in the values for these traces were obtained, which may indicate a reduction of the average radius of the pores, favoring the durability of the concrete.
\end{abstract}

Keywords: Nanomaterials, mechanical performance, water absorption. 
ORELLANA, J. P. L.; BRAGANÇA, M. O. G. P.; PORTELLA, K. F.; ALBERTI, E. L., NANOTECNOLOGIA NA CONSTRUÇÃO CIVIL: ESTUDO DO DESEMPENHO MECÂNICO E FÍSICO DE CONCRETOS COM ADIÇÃO DE NANOSÍLICA E NANOALUMINA COLOIDAIS. $4^{\circ}$ Simpósio Paranaense de Patologia das Construções (40 SPPC), artigo 4SPPC142, pp. 381 - 390, 2019.

\section{Introdução} DOI: $10.4322 / 2526-7248.060$

Novas tecnologias vêm chamando a atenção de vários pesquisadores pelo mundo todo: a incorporação de nanomateriais ao concreto. Dentre as nanoadições que estão sendo estudadas nos últimos anos, destacam-se alguns óxidos nanométricos, como é o caso do nano- $\mathrm{SiO}_{2}$ e do nano- $\mathrm{Al}_{2} \mathrm{O}_{3}$, mais conhecidos como nanosílica e nanoalumina, respectivamente. $\mathrm{O}$ nano- $\mathrm{SiO}_{2}$ parece ser o nanomaterial mais utilizado nas pesquisas [1], o qual pode atuar preenchendo os vazios entre os cristais de C-S$H$ [1], [2] e [3], e além disso, pela sua alta taxa de reação pozolânica, proporciona o potencial para a ocorrência de reações químicas com os produtos de hidratação do cimento [1]. Já o nano- $\mathrm{Al}_{2} \mathrm{O}_{3}$ não chega a ser tão usual quanto o anterior [1] e [3], mas a sua incorporação ao concreto conduz à formação do gel S-C-A (silicato de cálcio e alumínio), e também a reação com os cristais de hidróxido de cálcio, conferindo assim uma atividade pozolânica como no caso da nanosílica [1].

Autores como Behfarnia e Salemi (2013) [1] e Mohseni et al. (2015) [2] utilizaram estes dois materiais como adição ao concreto, e pesquisadores como Flores et al. (2017) [4] e Barbhuiya et al. (2014) [5] direcionaram os seus estudos a apenas uma das nanoadições, sendo a nanosílica e nanoalumina, respectivamente.

Foi constatado por Flores et al. (2017) [4] que a nanosílica promoveu um aumento significativo nas taxas de reação de hidratação da pasta e melhorou as propriedades mecânicas do concreto, principalmente nas primeiras idades. Já, Barbhuiya et al. (2014) [5] observaram que a nanoalumina atuou na densificação da microestrutura da pasta de cimento, reforçando que a mesma pode favorecer nas reações de hidratação do cimento. Sanchez et al. (2010) [3] direcionaram seus estudos a ambas as nanoadições e afirmaram que a nanosílica não se comportou apenas como um preenchedor dos vazios do concreto, mas, também, como um agente promissor de reações pozolânicas, e que o uso da nanoalumina aumentou o módulo de elasticidade de amostras com 5\% de adição em até 143\%. Behfarnia e Salemi (2013) [1] atribuíram também características de pozolanicidade à nanoalumina, as quais levaram ao aumento da densidade da matriz e a diminuição da presença de vazios na microestrutura do concreto.

A maioria dos estudos [1], [2], [3], [5] faz uso destes nanomateriais em pó, sendo necessário algum método de dispersão das nanopartículas, como por exemplo a mistura e agitação das mesmas em parte da água de amassamento e um aditivo superplastificante [1], [2]. Existe também a incorporação destes materiais em estado coloidal, como no caso de Flores et al. (2017) [4], no qual, é possível adicionar os mesmos diretamente ao concreto, sem a necessidade de dispersão prévia. Teores usuais estudados formam de 3\%, 5\% e 7\% [1]; 1\%, 3\%, e 5\% [2] para os autores que estudaram as duas nanoadições, e também foi possível observar 2,5\% [4] de nanosílica coloidal, e, $2 \%$ e $4 \%$ de nanoalumina em pó [5].

Assim, neste trabalho teve-se como objetivo avaliar as propriedades mecânicas e físicas de concretos com adição de nanosílica e nanoalumina, ambos em estado coloidal e em substituição de $1,5 \%$ em relação à massa de cimento, para verificar 0 comportamento deste tipo de materiais em estado coloidal, que não é tão explorado como o estado sólido (pó) destes. 
ORELLANA, J. P. L.; BRAGANÇA, M. O. G. P.; PORTELLA, K. F.; ALBERTI, E. L., NANOTECNOLOGIA NA CONSTRUÇÃO CIVIL: ESTUDO DO DESEMPENHO MECÂNICO E FÍSICO DE CONCRETOS COM ADIÇÃO DE NANOSÍLICA E NANOALUMINA COLOIDAIS. $4^{\circ}$ Simpósio Paranaense de Patologia das Construções (40 SPPC), artigo 4SPPC142, pp. 381 - 390, 2019.

\section{Materiais e métodos} DOI: $10.4322 / 2526-7248.060$

\subsection{Materiais}

O cimento utilizado neste estudo foi o CPIIZ-32 Votoran da empresa Votorantim Cimentos $L t d a$. Foi realizada uma caracterização física do mesmo em laboratório, de acordo com a NBR 16697 (2018) [6]. Como agregado miúdo foi utilizada uma areia artificial e como graúdo, uma brita com dimensão máxima de $25 \mathrm{~mm}$, ambos provenientes de uma pedreira localizada na região metropolitana de Curitiba. Foram realizadas, também, caracterizações físicas dos agregados em laboratório, conforme a NBR 7211 (2009) [7]. Para melhora da trabalhabilidade, foi utilizado um aditivo plastificante polifuncional redutor de água, fornecido pela empresa "GCP Applied Technologies". O aditivo foi o MIRA SET 27, cuja massa específica, conforme o fabricante, foi medida entre $1,150-1,210 \mathrm{~g} / \mathrm{cm}^{3}$.

Como adições, foram utilizadas a nanosílica (NSil), fornecida pela distribuidora Royal Marck Comercial Ltda, Levasil CB8 da marca AkzoNobel; e a nanoalumina (NAI), fornecida pela distribuidora Metachem Industrial e Comercial, da marca NYACOL Nano Technologies, Inc. Ambas as nanoadições apresentaram-se em estado coloidal. As informações fornecidas pelos fabricantes, em relação às propriedades dos materiais, encontram-se na Tabela 1.

Tabela 1: Resultados das caracterizações físicas e químicas dos nanomateriais fornecidos pelos respectivos fabricantes

\begin{tabular}{lcc}
\hline \multicolumn{1}{c}{ Parâmetro } & NSil & NAI \\
\hline Teor de $\mathrm{SiO}_{2}(\%)$ & 50 & - \\
Teor de $\mathrm{Al}_{2} \mathrm{O}_{3}(\%)$ & - & 20 \\
Massa específica $\left(\mathrm{g} / \mathrm{cm}^{3}\right)$ & 1,4 & 1,19 \\
Tamanho médio da partícula $(\mathrm{nm})$ & 35 & $60-90$ \\
$\mathrm{pH}$ & 9,5 & 4,0 \\
Aspecto/Cor da solução & Líquido branco & Líquido branco \\
Viscosidade $(\mathrm{cP})$ & 8 & $4-11$ \\
\hline
\end{tabular}

\subsection{Metodologia}

Neste estudo foi realizada a determinação do traço padrão a ser utilizado em todos os concretos. Para isto, foi definido que seriam dosados três traços, um traço sem adição e os outros dois com substituição de $1,5 \%$ de cada um dos nanomateriais apresentados na seção anterior, com relação à massa de cimento.

O estudo de dosagem foi feito pelo método de Furnas (1997), cujo traço padrão obtido foi de 1:2,07:2,90 e uma relação a/c de 0,564. O consumo de materiais para a confecção de $1 \mathrm{~m}^{3}$ de concreto está apresentado na Tabela 2.

O percentual de substituição de 1,5\% em relação ao cimento levou em consideração o teor de sólidos da NSil e da NAI, motivo pelo qual houve diferenças no consumo de cimento, das nanoadições e, também, da água de amassamento, na qual foi descontada a parte "não sólida" da solução coloidal destes nanomateriais. 
ORELLANA, J. P. L.; BRAGANÇA, M. O. G. P.; PORTELLA, K. F.; ALBERTI, E. L., NANOTECNOLOGIA NA CONSTRUÇÃO CIVIL: ESTUDO DO DESEMPENHO MECÂNICO E FÍSICO DE CONCRETOS COM ADIÇÃO DE NANOSÍLICA E NANOALUMINA COLOIDAIS. $4^{\circ}$ Simpósio Paranaense de Patologia das Construções (40 SPPC), artigo 4SPPC142, pp. 381 - 390, 2019. DOI: $10.4322 / 2526-7248.060$

Tabela 2: Valores relativos ao consumo de materiais por $\mathrm{m}^{3}$ de concreto (valores expressos em kg)

\begin{tabular}{cccccccc}
\hline Traço & Cimento & Areia & Brita & Água & Aditivo & NSil & NAL \\
\hline C-REF & 340 & 704 & 986 & 192 & 2,410 & - & - \\
C-NSil & 334 & 715 & 1001 & 189 & 1,570 & 11 & - \\
C-NAI & 317 & 711 & 995 & 179 & 1,807 & - & 26 \\
\hline
\end{tabular}

Após a determinação do traço, foi feita a moldagem dos corpos de provas para os ensaios mecânicos e físicos. Foram moldados cp's cilíndricos de $10 \mathrm{~cm}$ de diâmetro e $20 \mathrm{~cm}$ de altura $(10 \times 20) \mathrm{cm}$, e foram realizadas as caracterizações dos diferentes traços de concreto no estado fresco pelo abatimento do tronco de cone (slump test) e pela massa unitária. Finalmente, foi realizada a caracterização dos concretos no estado endurecido por meio de ensaios mecânicos e ensaios físicos de absorção, conforme apresentados na Tabela 3.

Tabela 3: Descritivo dos ensaios normatizados para a determinação de parâmetros mecânicos e físicos dos concretos de cimento Portland com traços com nanomateriais e referência, no estado endurecido

\begin{tabular}{lcc}
\hline \multicolumn{1}{c}{ Ensaio } & Norma & Idade \\
\hline & NBR 5739 [8] & 7 dias \\
$\begin{array}{l}\text { Resistência à compressão axial } \\
\text { Resistência à tração por }\end{array}$ & dias \\
$\begin{array}{l}\text { compressão diametral } \\
\text { Módulo de elasticidade estático }\end{array}$ & NBR 8522 [9] [10] & 28 dias \\
$\begin{array}{l}\text { Absorção de água, índice de } \\
\text { vazios e massa específica real }\end{array}$ & NBR 9778 [11] & 28 dias \\
Absorção de água por capilaridade & NBR 9779 [12] & 28 dias \\
\hline
\end{tabular}

\section{Resultados e discussões}

Os resultados da caracterização do cimento Portland CPIIZ-32 Votoran estão apresentados na Tabela 4.

Tabela 4: Caracterização física do cimento realizada em laboratório

\begin{tabular}{lc}
\hline \multicolumn{1}{c}{ Ensaio de caracterização } & Resultado \\
\hline Massa específica $\left(\mathrm{g} / \mathrm{cm}^{3}\right)$ & 3,05 \\
Tempo de início de pega $(\mathrm{min})$ & 260 \\
Tempo de final de pega $(\mathrm{min})$ & 360 \\
Expansibilidade a quente $(\mathrm{mm})$ & 0,0 \\
Consistência normal $(\%)$ & 27,6 \\
Finura pelo método de Blaine $\left(\mathrm{cm}^{2} / \mathrm{g}\right)$ & 3680 \\
Índice de finura por meio de peneirador & \\
aerodinâmico na peneira de $75 \mu \mathrm{m}(\%)$ & 2,3 \\
Índice de finura por meio de peneirador & \\
aerodinâmico na peneira de 45 $4 \mathrm{~m}(\%)$ & 9,6 \\
\hline
\end{tabular}


ORELLANA, J. P. L.; BRAGANÇA, M. O. G. P.; PORTELLA, K. F.; ALBERTI, E. L., NANOTECNOLOGIA NA CONSTRUÇÃO CIVIL: ESTUDO DO DESEMPENHO MECÂNICO E FÍSICO DE CONCRETOS COM ADIÇÃO DE NANOSÍLICA E NANOALUMINA COLOIDAIS. $4^{\circ}$ Simpósio Paranaense de Patologia das Construções (40 SPPC), artigo 4SPPC142, pp. 381 - 390, 2019. DOI: $10.4322 / 2526-7248.060$

Por meio da Tabela 4, é possível concluir que os parâmetros físicos avaliados no cimento atendem aos limites especificados pela NBR 16697 (2008) [6].

Os resultados da caracterização física dos agregados miúdos e graúdos estão mostrados na Tabela 5.

Tabela 5: Resultados da caracterização dos agregados miúdo e graúdo realizada em laboratório

\begin{tabular}{lcc}
\hline \multirow{2}{*}{ Ensaio de caracterização } & \multicolumn{2}{c}{ Resultados } \\
\cline { 2 - 3 } & $\begin{array}{c}\text { Agregado } \\
\text { miúdo }\end{array}$ & $\begin{array}{c}\text { Agregado } \\
\text { graúdo }\end{array}$ \\
\hline Módulo de finura & 2,96 & 7,02 \\
Dimensão Máxima Característica $(\mathrm{mm})$ & 4,8 & 25 \\
Massa específica $\left(\mathrm{g} / \mathrm{cm}^{3}\right)$ & 2,58 & 2,63 \\
Massa unitária $\left(\mathrm{kg} / \mathrm{m}^{3}\right)$ & 1570 & 1420 \\
Absorção de água $(\%)$ & 0,3 & 0,6 \\
Volume de vazios $(\%)$ & 39,1 & 45,9 \\
Material pulverulento $(\%)$ & 7,4 & 0,4 \\
Índice de forma & - & 2,8 \\
\hline
\end{tabular}

Segundo a NBR 7211 (2009) [7] os resultados apresentados na Tabela 5 foram condizentes com os objetivos propostos para a realização dos traços de concreto.

Os resultados da caracterização dos diferentes traços de concreto no estado fresco, realizados pelo abatimento em tronco de cone e pela massa unitária, estão apresentados na Tabela 6.

Tabela 6: Resultados da caracterização dos concretos no estado fresco, quanto ao abatimento em tronco de cone e de massa unitária

\begin{tabular}{lccc}
\hline \multicolumn{1}{c}{ Ensaio } & \multicolumn{3}{c}{ Resultado } \\
\cline { 2 - 4 } & C-REF & C-NSil & C-NAL \\
\hline Abatimento $(\mathrm{mm})$ & 120 & 100 & 95 \\
Massa unitária $\left(\mathrm{kg} / \mathrm{m}^{3}\right)$ & 2217 & 2239 & 2222 \\
\hline
\end{tabular}

Foi estipulado um abatimento de $110 \pm 10 \mathrm{~mm}$. Por meio da Tabela 6 , foi possível concluir que o C-REF obteve uma maior fluidez com relação aos traços com nanomaterial incorporado. Este resultado é esperado devido ao efeito da elevada superfície específica das nanoadições, que em consequência reduz a fluidez do concreto, aumentando assim a coesão.

Os resultados das caracterizações mecânicas dos três traços de concreto realizados, pelos ensaios de resistência à compressão axial, resistência à tração por compressão diametral e pelo módulo de elasticidade estático estão apresentados na Figura 1, na Figura 2 e na Figura 3, respectivamente. 
ORELLANA, J. P. L.; BRAGANÇA, M. O. G. P.; PORTELLA, K. F.; ALBERTI, E. L., NANOTECNOLOGIA NA CONSTRUÇÃO CIVIL: ESTUDO DO DESEMPENHO MECÂNICO E FÍSICO DE CONCRETOS COM ADIÇÃO DE NANOSÍLICA E NANOALUMINA COLOIDAIS. $4^{\circ}$ Simpósio Paranaense de Patologia das Construções (40 SPPC), artigo 4SPPC142, pp. 381 - 390, 2019. DOI: $10.4322 / 2526-7248.060$

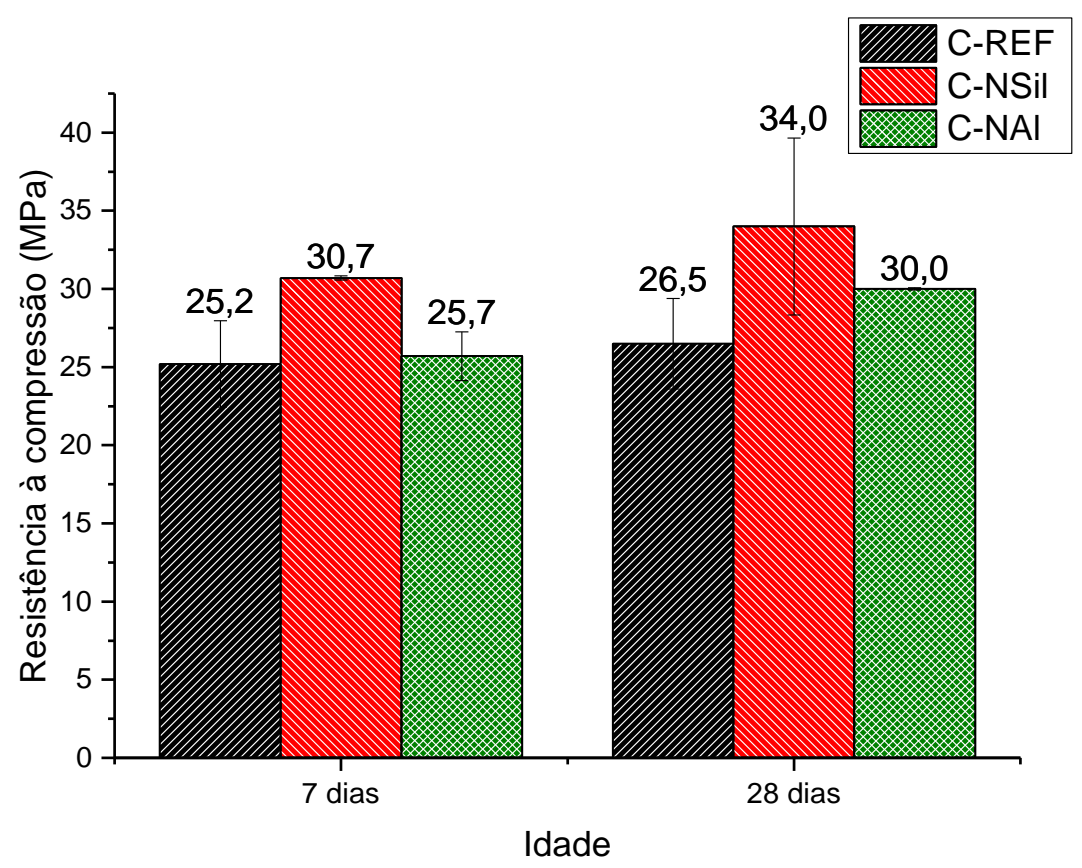

Figura 1: Gráficos do tipo barra da resistência à compressão axial dos três traços de concreto de cimento Portland, em função do tempo de cura úmida.

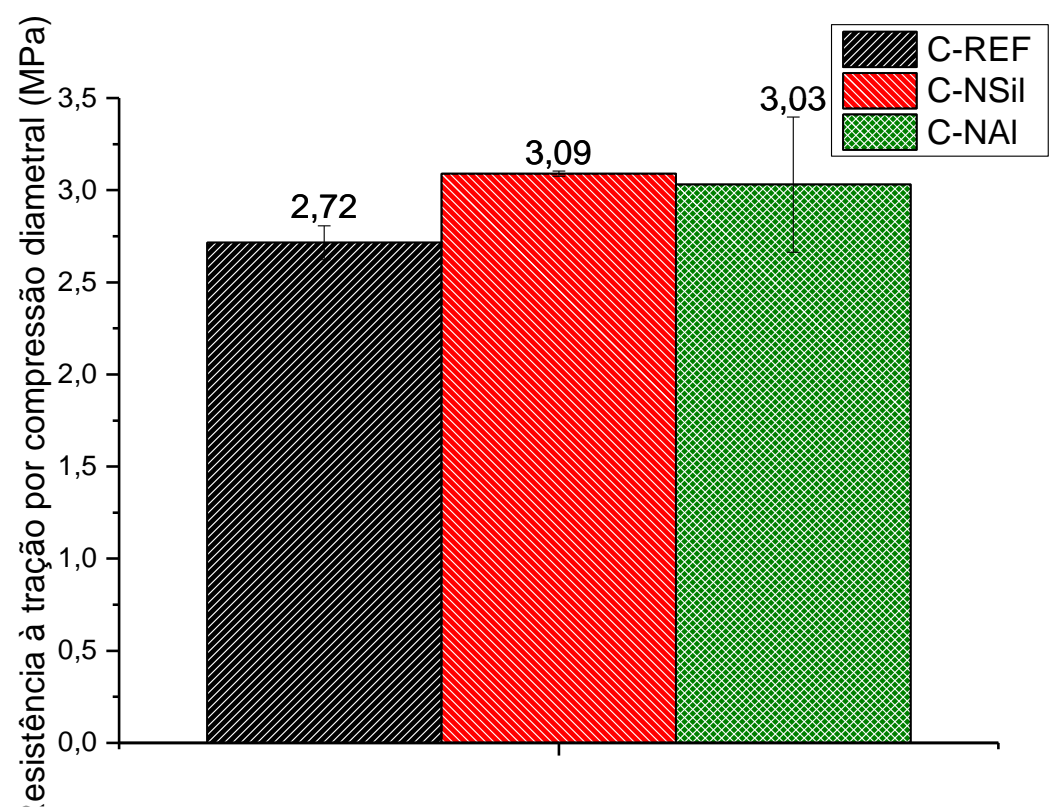

Figura 2: Gráficos do tipo barra da resistência à tração por compressão diametral dos três traços de concreto de cimento Portland, aos 28 dias de cura úmida. 


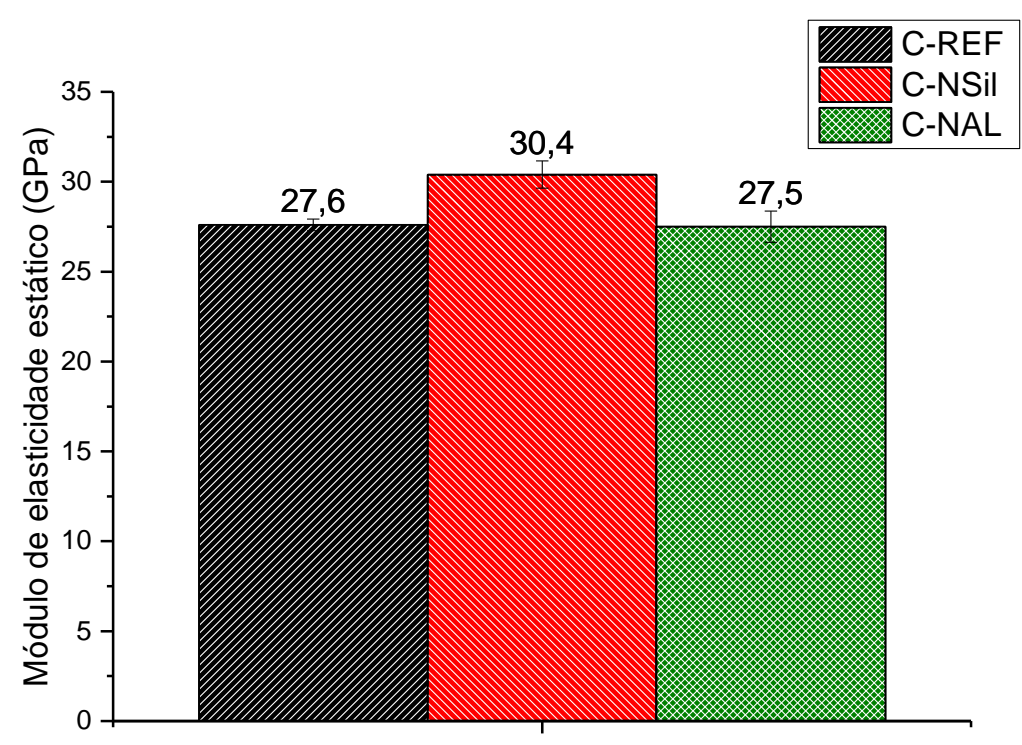

Figura 3: Gráficos do tipo barra do módulo de elasticidade estático dos concretos, aos 28 dias de cura úmida.

Dos resultados mecânicos apresentados foi possível observar um aumento nas três propriedades avaliadas com o uso dos nanomateriais, com exceção do módulo de elasticidade e da resistência à compressão aos 7 dias para o traço C-NAl, nos quais não houve uma diferença significativa com relação ao C-REF. A NSil, no entanto, apresentou melhorias em todos os ensaios mecânicos avaliados, destaque para a resistência à compressão aos 28 dias, que apresentou um aumento de $28,3 \%$ com relação ao traço sem adição. No entanto, é importante observar a variabilidade do resultado para este traço aos 28 dias, pela barra de erro, que apresenta-se bem superior se comparada aos demais traços. Estes resultados reforçam ainda mais 0 indicado por [1], [2], [3] e [4], de que a nanosílica atua no preenchimento dos vazios do concreto, e ainda mais, possui uma atividade pozolânica, que leva à formação dos cristais de C-S-H no lugar do hidróxido de cálcio, sendo esses responsáveis pela resistência mecânica do concreto.

Os resultados estão de acordo com os obtidos por Behfarnia e Salemi (2013) [1], que estudaram também concretos com nanosílica e nanoalumina, os quais foram os traços com a primeira que apresentaram os melhores desempenhos à compressão. O resultado de resistência à compressão do traço C-NAl também corroborou com o de Barbhuiya et al. (2014) [5], que nas primeiras idades (até 7 dias) não verificaram melhoria com relação ao traço sem adição, já que isso pode ser atribuído ao fato de que estes não encontraram nenhuma nova fase cristalina pelo DRX até os 7 dias de idade. Com relação ao módulo de elasticidade, no entanto, o resultado do traço CNAl não esteve de acordo com Sanchez et al. (2010) [3], que afirmaram que o concreto com nanoalumina aumentou em $143 \%$ o módulo de elasticidade. Isso pode ser atribuído ao fato do teor da adição, que no caso dos autores foi de $5 \%$, e neste estudo o teor foi de apenas 1,5\%, com relação à massa de cimento, e também a forma em que a mesma foi incorporada, sendo no estudo dos autores em pó, e neste estudo em estado coloidal. Estes afirmaram também que a nanoalumina apresentou um efeito limitado na resistência à compressão, pois a mesma não aumenta significativamente com a adição do material em questão, principalmente nas primeiras idades, como foi possível observar também neste trabalho. 
ORELLANA, J. P. L.; BRAGANÇA, M. O. G. P.; PORTELLA, K. F.; ALBERTI, E. L., NANOTECNOLOGIA NA CONSTRUÇÃO CIVIL: ESTUDO DO DESEMPENHO MECÂNICO E FÍSICO DE CONCRETOS COM ADIÇÃO DE NANOSÍLICA E NANOALUMINA COLOIDAIS. $4^{\circ}$ Simpósio Paranaense de Patologia das Construções (40 SPPC), artigo 4SPPC142, pp. 381 - 390, 2019. DOI: $10.4322 / 2526-7248.060$

Os resultados de absorção de água por imersão e absorção por capilaridade estão apresentados na Figura 4 e Figura 5, respectivamente.

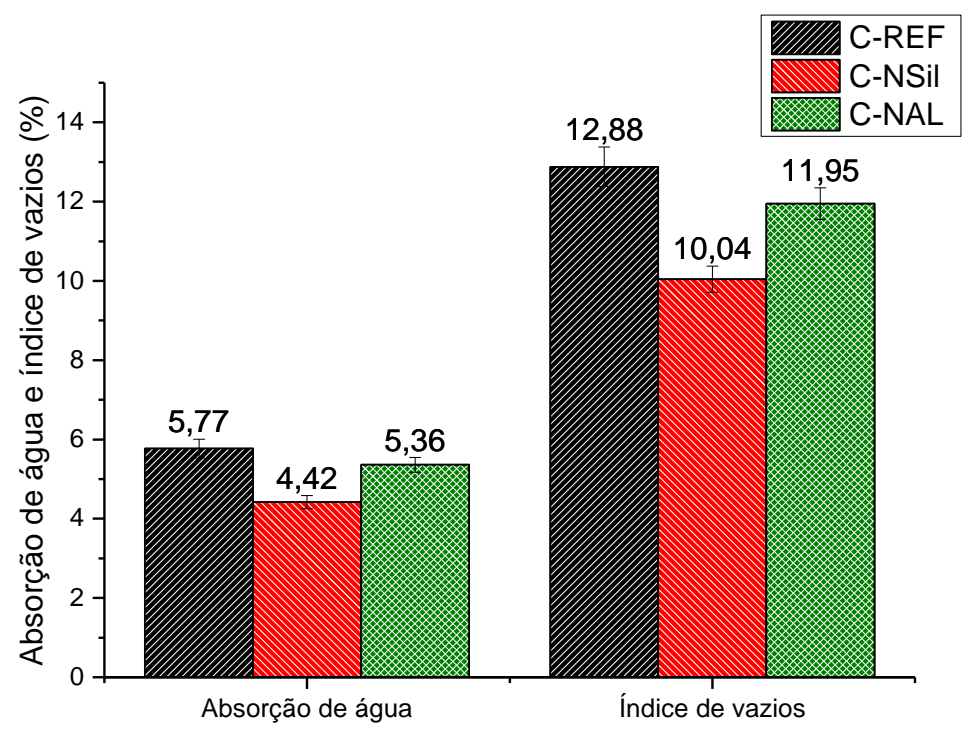

Figura 4: Gráficos do tipo barra do coeficiente de absorção por imersão e índice de vazios dos três traços de concreto de cimento Portland, aos 28 dias.

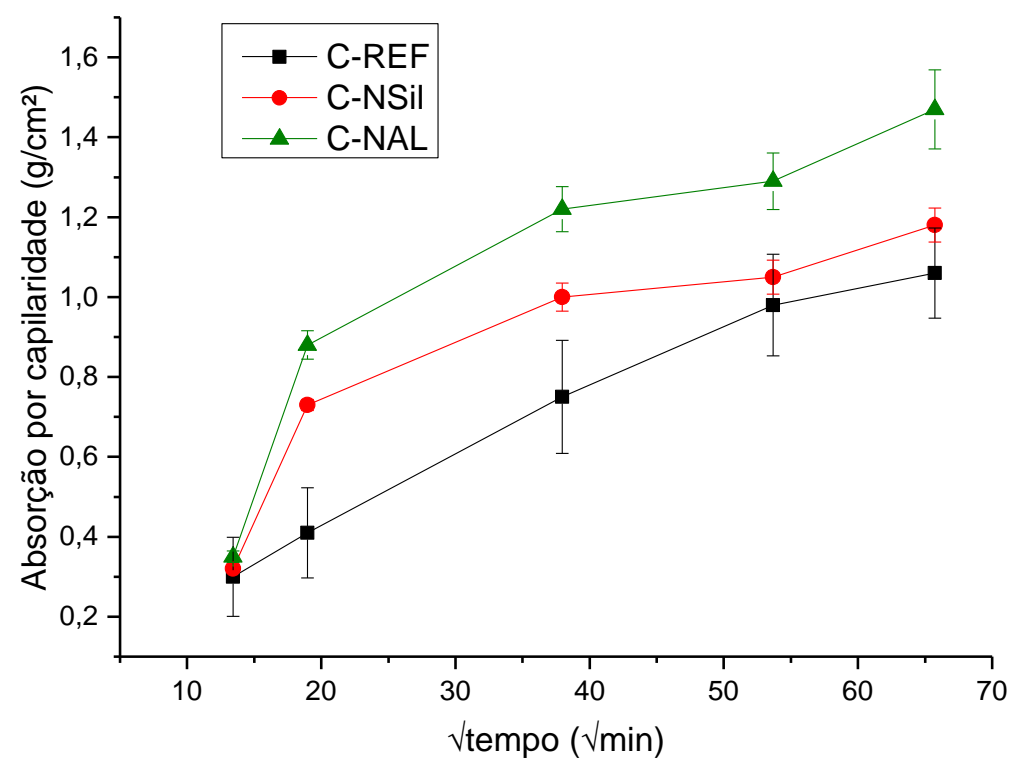

Figura 5: Gráficos do tipo linha da absorção de água por capilaridade dos três traços de concreto de cimento Portland, em função do tempo de ensaio.

Dos ensaios físicos, foi possível concluir que a incorporação das nanoadições reduziu os valores de absorção de água e do índice de vazios em $23,4 \%$ e $22 \%$, respectivamente. Este fato foi observado também por Behfarnia e Salemi (2013) [1], cujo estudo apontou também para a redução da absorção de água em concretos com nanosílica e nanoalumina, assim como por Mohseni et al. (2015) [2], com destaque para a nanosílica, que apresentou o menor valor dentre os traços estudados pelos autores. Estes resultados corroboraram assim com o presente estudo. Os valores inferiores de absorção e índice de vazios foram indicativos de redução da porosidade 
ORELLANA, J. P. L.; BRAGANÇA, M. O. G. P.; PORTELLA, K. F.; ALBERTI, E. L., NANOTECNOLOGIA NA CONSTRUÇÃO CIVIL: ESTUDO DO DESEMPENHO MECÂNICO E FÍSICO DE CONCRETOS COM ADIÇÃO DE NANOSÍLICA E NANOALUMINA

COLOIDAIS. $4^{\circ}$ Simpósio Paranaense de Patologia das Construções (40 SPPC), artigo 4SPPC142, pp. 381 - 390, 2019. DOI: $10.4322 / 2526-7248.060$

do concreto, o que corroborou com os testes mecânicos realizados, nos quais o CNSil apresentou melhores desempenhos. Assim, estes resultados reforçam o efeito de preenchimento dos vazios do concreto pelos nanomateriais incorporados, assim como os resultados mecânicos.

Já, a absorção por capilaridade, foi representada em função da raiz quadrada do tempo, assim como feito por Macedo et al. (2016) [13]. O traço com os menores valores de absorção por capilaridade foi o C-REF. Segundo os autores [13], este fato pode ser atribuído ao menor raio médio dos poros, que elevou os percentuais de absorção capilar nos traços com NSil e NAl, pois o raio médio dos poros é inversamente proporcional à altura de ascensão capilar, e esta está diretamente ligada ao volume de água absorvido no ensaio [13]. Como o refinamento dos poros é um aspecto favorável ao aumento da durabilidade do concreto, a incorporação destes nanomateriais no concreto pode ser um bom indicativo do aumento da vida útil dessas estruturas. No entanto, os resultados deste ensaio não corroboraram com os outros ensaios, pois o traço com maior absorção foi o C-NAl, sendo que este obteve o desempenho intermediário no ensaio de absorção por imersão.

\section{Conclusões}

A incorporação de nanomateriais ao concreto, como a NSil e a NAI, mostrou ser eficiente ao melhorar e manter, em alguns casos, as propriedades mecânicas e físicas do concreto.

As propriedades mecânicas foram melhoradas, principalmente no traço que contém NSil, destaque para o incremento na resistência à compressão aos 28 dias, que chegou a quase $30 \%$. No módulo de elasticidade de estático, no entanto, não houve diferença significativa do traço C-NAL com o C-REF.

As propriedades físicas de absorção de água também foram aprimoradas com as nanoadições. O C-NSil foi o que teve valores de absorção de água por imersão e índice de vazios menores dentre todos os traços, cujos valores foram de $4,42 \%$ e $10,04 \%$, respectivamente, demonstrando uma redução de cerca de $25 \%$ em relação ao concreto sem adição, o que é um indicativo de que a porosidade total deste concreto é menor dentre os demais. Na absorção por capilaridade, o traço sem adição foi o que teve os menores valores, chegando a $1,06 \mathrm{~g} / \mathrm{cm}^{2}$ às 72 horas de ensaio.

\section{Agradecimentos}

Ao Lactec, pela infraestrutura disponibilizada; à Universidade Federal do Paraná, em especial ao Programa de Pós-Graduação em Engenharia de Construção Civil (PPGECC), no qual está em andamento o mestrado do primeiro autor; também à ANEEL e às Centrais Elétricas do Rio Jordão (Elejor) pelo projeto de pesquisa P\&D ANEEL, P\&D 2945-01000/2017 no qual está inserido este estudo; e ao CNPq DT, processo número 302672/2016.

Agradecer também à Votorantim Cimentos Ltda, Royal Marck Comercial Ltda, e à Metachem Industrial e Comercial pela doação do cimento, NSil e NAl, respectivamente, pela qual foi possível a confecção dos corpos de prova para os testes efetuados. 
ORELLANA, J. P. L.; BRAGANÇA, M. O. G. P.; PORTELLA, K. F.; ALBERTI, E. L., NANOTECNOLOGIA NA CONSTRUÇÃO CIVIL: ESTUDO DO DESEMPENHO MECÂNICO E FÍSICO DE CONCRETOS COM ADIÇÃO DE NANOSÍLICA E NANOALUMINA COLOIDAIS. $4^{\circ}$ Simpósio Paranaense de Patologia das Construções (40 SPPC), artigo 4SPPC142, pp. 381 - 390, 2019.

\section{Referências} DOI: $10.4322 / 2526-7248.060$

[1] Behfarnia, K.; Salemi, N. (2013) The effects of nano-silica and nano-alumina on frost resistance of normal concrete, Construction and Building Materials, 48: 580584.

[2] Mohseni, E.; Miyandehi, B. M.; Yang, J.; Yadzi, M. A. (2015) Single and combined effects of nano- $\mathrm{SiO}_{2}$, nano- $\mathrm{Al}_{2} \mathrm{O}_{3}$ and nano- $\mathrm{TiO}_{2}$ on the mechanical, rheological and durability properties of self-compacting mortar containing fly ash, Construction and Building Materials, 84: 331-340.

[3] Sanchez, F.; Sobolev, K. (2010) Nanotechnology in concrete - A review, Construction and Building Materials, 24: 2060-2071.

[4] Flores, Y. C.; Cordeiro, G. C.; Toledo Filho, R. D.; Tavares, L. M. (2017) Performance of Portland cement pastes containing nano-silica and different types of silica, Construction and Building Materials, 146: 524-530.

[5] Barbhuiya, S.; Mukherjee, S.; Nikraz, H. (2014) Effects of nano- $\mathrm{Al}_{2} \mathrm{O}_{3}$ on early-age microstructural properties of cement paste, Construction and Building Materials, 52: 189-193.

[6] NBR 16697 (2018) Cimento Portland - Requisitos. Associação Brasileira de Normas Técnicas - ABNT, Rio de Janeiro, Brasil.

[7] NBR 7211 (2009) Agregados para concreto - Especificação. Associação Brasileira de Normas Técnicas - ABNT, Rio de Janeiro, Brasil.

[8] NBR 5739 (2018) Concreto - Ensaio de compressão de corpos-de-prova cilíndricos. Associação Brasileira de Normas Técnicas - ABNT, Rio de Janeiro, Brasil.

[9] NBR 7222 (2011) Concreto e argamassa - Determinação da resistência à tração por compressão diametral de corpos de prova cilíndricos. Associação Brasileira de Normas Técnicas - ABNT, Rio de Janeiro, Brasil.

[10] NBR 8522 (2017) Concreto - Determinação do módulo de elasticidade estático à compressão. Associação Brasileira de Normas Técnicas - ABNT, Rio de Janeiro, Brasil.

[11] NBR 9778 (2005) Argamassa e concreto endurecidos - Determinação da absorção de água, índice de vazios e massa específica. Associação Brasileira de Normas Técnicas - ABNT, Rio de Janeiro, Brasil.

[12] NBR 9779 (2012) Argamassa e concreto endurecidos - Determinação da absorção de água por capilaridade. Associação Brasileira de Normas Técnicas - ABNT, Rio de Janeiro, Brasil.

[13] Macedo, R. C.; Silva, P .H. V.; Souza, T. C. C.; Calixto, J. M. F. (2016) Avaliação da porosidade e da hidratação de argamassas com nanotubos de carbono sintetizados diretamente sobre clínquer de cimento Portland, Congresso Brasileiro de Concreto - 58 CBC2016, Belo Horizonte, MG, Brasil. 